\title{
Demethylation of microRNA-142 induced by demethylation agents plays a suppressive role in osteosarcoma cells
}

\author{
MULIANG DING ${ }^{1}$, JIANZHONG HU $^{1}$, JIANGDONG NI ${ }^{2}$, ZHONGHUI ZHENG $^{2}$, DEYE SONG $^{2}$ and JUNJIE WANG ${ }^{2}$ \\ ${ }^{1}$ Department of Orthopedics, Xiangya Hospital; ${ }^{2}$ Department of Orthopedics, The Second Xiangya Hospital, \\ Central South University, Changsha, Hunan 410008, P.R. China
}

Received June 18, 2014; Accepted February 11, 2015

DOI: 10.3892/ol.2015.3036

\begin{abstract}
Osteosarcoma (OS), the most common malignant bone tumor, occurs mainly in adolescents and young adults, with a morbidity of $\sim 5$ cases per million. The expression levels of microRNAs (miRNAs) as tumor suppressors were recently found to be downregulated in OS. Certain alterations of miRNAs and the possible mechanisms through which miRNAs affect cell proliferation and migration in OS were recently found to be correlated with methylation epigenetic mechanisms. In this study, it was demonstrated that, due to hypermethylation, the expression level of miRNA-142 (miR-142) was significantly downregulated in OS tissues and cells compared with that in control samples. The present study demonstrated an increased expression of miR-142 in Saos-2 and MG63 cells treated with demethylation agents, suggesting that the effect of such agents on cell growth, inhibition of invasion and cell cycle retardation may be mediated by miR-142 in OS.
\end{abstract}

\section{Introduction}

Osteosarcoma (OS), the most common malignant bone tumor, occurs mainly in adolescents and young adults, with a morbidity of $\sim 5$ cases per million (1). OS develops from a common mesenchymal stem cell progenitor exhibiting a disruption of normal osteoblast differentiation (2). Although the 5-year survival rate has increased to $60-70 \%$, a significant proportion of patients respond poorly to chemotherapy and are at high risk of relapse or metastasis, even following curative resection (3). The majority of OSs exhibit numerous and highly variable genomic aberrations. DNA methylation is an important mechanism for the downregulation of gene expression, more specifically at $\mathrm{CpG}$ islands in the promoter region of genes. It was previously reported that epigenetic modifica-

Correspondence to: Professor Jianzhong $\mathrm{Hu}$, Department of Orthopedics, Xiangya Hospital, Central South University, 87 Xiang-ya Road, Changsha, Hunan 410008, P.R. China

E-mail: hjzdocxy@gmail.com; hujianzhong2014@163.com

Key words: osteosarcoma, methylation, microRNA, tumor suppressor tions may be more prevalent than mutations in certain types of childhood cancer, such as retinoblastoma (4). A number of study groups have reported comparisons of alterations in DNA methylation/deacetylation and microRNA (miRNA) expression for other types of cancer (5), although only a limited number of studies have investigated the association of these types of mechanisms with OS. The identification of molecular markers and pathways contributing to OS development and progression may facilitate earlier diagnosis and the development of novel treatment strategies.

miRNAs are short endogenous non-coding RNAs that post-transcriptionally regulate the expression of target genes (oncogenes or tumor suppressor genes) involved in several types of cancer, including OS (6). A single miRNA may silence a large number of genes, allowing these molecules extensive control over numerous cellular functions (7). Evidence of individual miRNAs affecting developmental biology, cellular differentiation and oncogenesis is continuously emerging (8). Of note, it was recently reported that miRNAs may be able to cause heritable changes in gene expression by epigenetic mechanisms, such as altering DNA methyltransferase levels, whereas miRNAs themselves may be regulated by epigenetic mechanisms (9). Certain alterations in miRNAs and the possible mechanisms through which miRNAs affect cell proliferation and migration in OS were recently found to be correlated with methylation epigenetic mechanisms $(10,11)$. The majority of the downregulated miRNAs were found to be silenced through epigenetic mechanisms in solid malignancies, including breast cancer (12) and neuroblastoma (13). In OS, the expression levels of tumor suppressor miRNAs, including miRNA-34 (miR-34) and miR-143, were recently found to be downregulated. The miR-34 gene was hypermethylated in OS, which was found to be associated with cancer cell metastasis (14). However, there is currently no sufficient evidence supporting the correlation of miRNA overexpression in OS with epigenetic events. It was suggested that miR-142 may play an important role in maintaining the self-renewal capacity of bronchioalveolar stem cells (15). It was also reported that patients with esophageal squamous cell carcinoma (ESCC) displaying a high expression of miR-142 had poorer survival rates compared with those with low expression of miR-142, suggesting that miR-142 may act as a tumor suppressor (16).

Although miR-142 has been found to be downregulated in OS cell lines (17), the role and epigenetic mechanisms of 
miR-142 in OS remain unknown. The aim of the present study was to compare the expression of miR-142 between OS and pericancerous tissues and investigate the role of epigenetic regulation in the decreased expression of miR-142 in OS.

\section{Materials and methods}

Tissue samples. All the patients signed an informed consent, approved by the Independent Ethics Committee of Central South University (Changsha, China). The OS and matched adjacent normal tissues used in this study were collected from 6 patients who were treated between 2011 and 2014 in Xiangya Hospital (Changsha, China). All the samples were stored at $-80^{\circ} \mathrm{C}$ until tissue analysis.

Cell culture. hFOB1.19, Saos-2, MG63, U2OS and HOS cells were obtained from American Type Culture Collection (Manassas, VA,USA). All the cells were cultured in RPMI-1640 basic medium (Gibco Life Technologies, Carlsbad, CA, USA) supplemented with $10 \%$ fetal bovine serum (FBS; Invitrogen Life Technologies, Carlsbad, CA, USA). All the cells were cultured under conditions of $95 \%$ air and $5 \% \mathrm{CO}_{2}$ at $37^{\circ} \mathrm{C}$.

Epigenetic drug treatment of cells. The Saos-2 and MG63 cells were divided in four groups as follows: i) Control cells; ii) cells treated with $15.55 \mathrm{nM}$ of the demethylation drug 5-Aza-2'-deoxycytidine (Aza; Sigma-Aldrich, St. Louis, MO, USA); iii) cells treated with $1.5 \mathrm{nM}$ of the histone deacetylase inhibitor 4-phenylbutyric acid (PBA; Sigma-Aldrich); and iv) cells co-treated with $15.55 \mathrm{nM}$ Aza and 1.5 nM PBA (Aza + PBA).

Quantitative polymerase chain reaction ( $q P C R)$ analysis. Total RNA was extracted from the indicated cells using TRIzol ${ }^{\circledR}$ reagent (Invitrogen Life Technologies) following the manufacturer's instructions. The specific primers for miRNA-142 and U6 were purchased from GeneCopoeia (Rockville, MD, USA). The relative expression of miR-142 was measured using the miScript SYBR ${ }^{\circledR}$ Green PCR Kit (Qiagen, Venlo, The Netherlands). The expression of U6 was used as an endogenous control. Data were processed using the $2^{-\Delta \Delta \mathrm{Ct}}$ method.

Cell counting kit-8 (CCK-8) cell proliferation assay. CCK-8 was used to evaluate cell proliferation. A total of $5 \times 10^{3}$ cells were seeded in each 96 -well plate for $24 \mathrm{~h}$, treated with the indicated drugs and further incubated for $0,24,48$ and $72 \mathrm{~h}$. One hour prior to the completion of the incubation, $10 \mu \mathrm{l}$ CCK -8 reagent was added to each well. The optical density (OD) $450 \mathrm{~nm}$ value in each well was determined by an enzyme immunoassay analyzer (Thermo Fisher Scientific, Inc., Waltham, MA, USA).

Transwell ${ }^{\mathrm{TM}}$ assay. Following treatment with the indicated drugs for $72 \mathrm{~h}$, the cells were starved in serum-free medium (Invitrogen Life Technologies) for $24 \mathrm{~h}$ and resuspended in serum-free medium. The cells were added to the upper chamber, while the lower chamber was filled with basic medium containing 10\% FBS. Following incubation for $24 \mathrm{~h}$, the cells attached to the bottom were fixed and stained with crystal violet (Amresco, LLC, Solon, OH, USA) for $20 \mathrm{~min}$. The redundant crystal violet was washed by $0.1 \mathrm{M}$ phosphate-buffered saline (PBS; Amresco, LLC), and dried in air. The OD of crystal violet dissolved in $10 \%$ acetic acid at $570 \mathrm{~nm}$ was detected by an enzyme immunoassay analyzer.

Flow cytometric analysis of the cell cycle. The cells were treated with the indicated drugs for $72 \mathrm{~h}$. Following trypsinization and washing with ice-cold PBS, the cell suspensions were stained using BD Cycletest ${ }^{\mathrm{TM}}$ Plus (BD Biosciences, Franklin Lakes, NJ, USA) according to the manufacturer's instructions and the cell cycle was then analyzed by flow cytometry (Beckman Coulter, Brea, CA, USA). The experiments were performed in triplicate.

Measurement of miR-142 promoter CpG island methylation status by bisulfite genomic sequencing PCR (BSP) and methylation-specific PCR (MSP). Genomic DNA was extracted using the Takara Genomic DNA Extraction kit (Takara Co., Dalian, China). Genomic DNA (1 $\mu \mathrm{g}$ per sample) was modified with bisulfite using the Epitect Bisulfite kit (Qiagen) according to the manufacturer's instructions. The modified DNA was amplified using the following primers: miR-142 forward, TTTAGGGTATGAGAGAGGGTTTTTAG and reverse, AATTCCCTTCAAAAAAAACAAAACT. The PCR products were gel-extracted (Qiagen) to confirm that a single band had been obtained and were then sequenced by Invitrogen Life Technologies.

MSP was performed on bisulfate-treated DNA. The primers used were as follows: Unmethylated miR-142 forward, TTAAGGTTTGTGTGGGTTTAAATG and reverse, AAATAAACAAAAAACAACATCAAC; methylated miR-142 forward, TTTTAAGGTTTGTGTGGGTTTAAAC and reverse, AAAATAAACAAAAAACAACGTCGAC. The annealing temperature was $60^{\circ} \mathrm{C}$ for methylated-PCR and $55^{\circ} \mathrm{C}$ for unmethylated-PCR, with 27 cycles used for each.

Statistical analysis. Student's t-tests or one-way analysis of variance were used to analyze data using GraphPad Prism 5 software (GraphPad Software, Inc., La Jolla, CA, USA), depending on the experimental conditions. All the data are presented as mean \pm standard deviation. Compared with the respective controls, $\mathrm{P}<0.05$ was considered to indicate a statistically significant difference.

\section{Results}

Methylation of CpG islands and expression of $m i R-142$ in OS tissues and cell lines. The average expression level of miR-142 was found to be significantly lower $(\mathrm{P}<0.001)$ in the OS samples obtained from the 6 OS patients compared with the normal controls, as indicated by the reverse transcription-qPCR (Fig. 1A). Similar results were observed in the OS cell lines, particularly in Saos-2 and MG63 cells (Fig. 1B). In light of these findings, the role of epigenetic mechanisms that may be involved in the silencing of miR-142 was investigated. MSP was used to detect miR-142 methylation in tissues obtained from the 6 patients with OS and the normal tissue samples (Fig. 1C). A significantly higher level of methylation was observed in OS compared with that in 
A

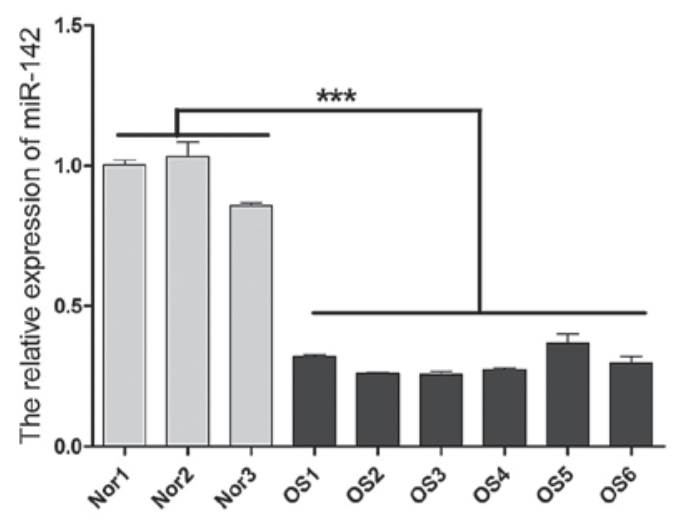

B

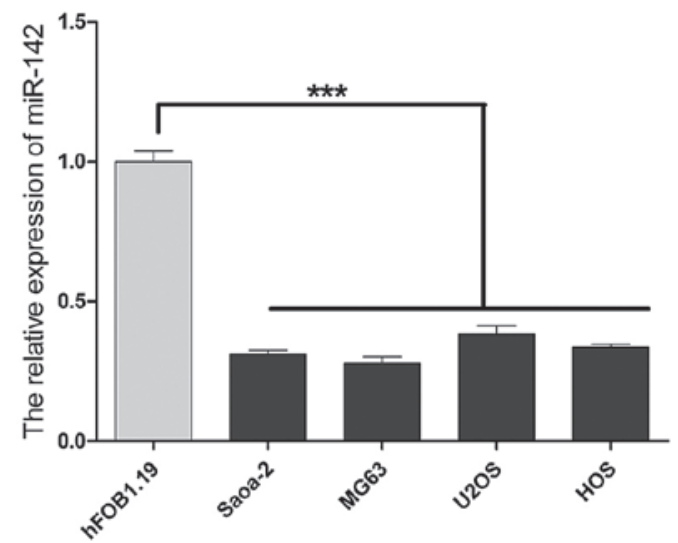

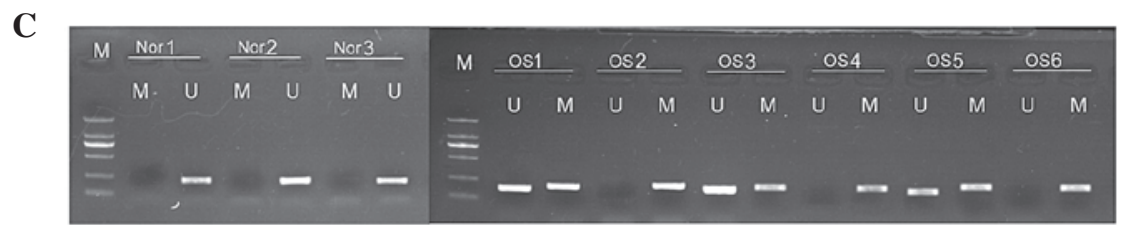

Figure 1. MicroRNA (miR)-142 expression and CpG island methylation. (A) The miR-142 expression levels were significantly lower in OS tissues compared to those in normal tissues. (B) The miR-142 expression levels were significantly lower in OS cell lines compared to those in hFOB1.19 controls (C) Methylation-specific polymerase chain reaction test results for the 6 OS patients and the 3 normal controls. Data are expressed as mean \pm standard deviation. ${ }^{* * * *} \mathrm{P}<0.001$ vs. control.

A

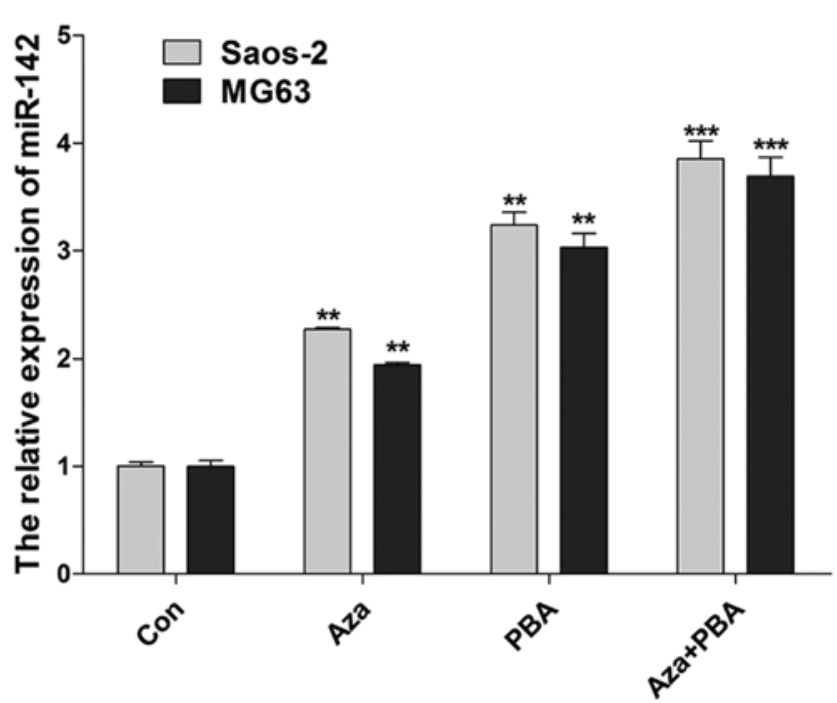

B

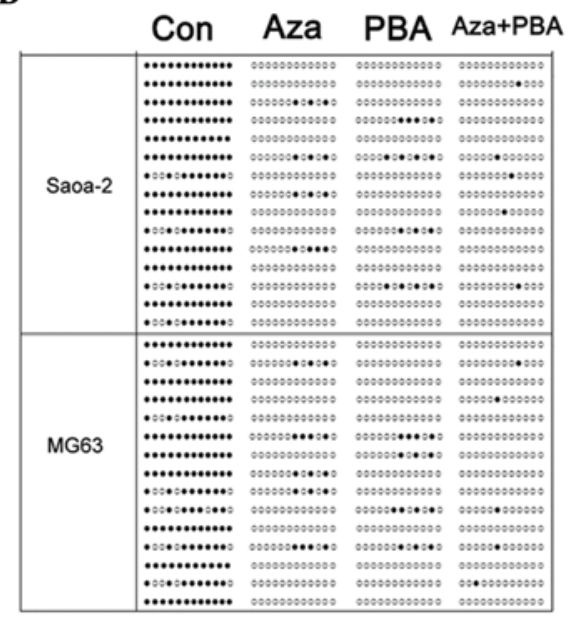

Figure 2. MicroRNA (miR)-142 expression and CpG island methylation following treatment with demethylation agents. (A) The miR-142 expression levels were significantly increased by demethylation agents in Saos-2 and MG63 cells. (B) The bisulfite genomic sequencing polymerase chain reaction detection results demonstrated that methylation was decreased following treatment. Data are expressed as mean \pm standard deviation. ${ }^{* * *} \mathrm{P}<0.01$ and ${ }^{* * * *} \mathrm{P}<0.001$ vs. control (Con). Aza, 5-Aza-2'-deoxycytidine; PBA, 4-phenylbutyric acid.

normal tissues. There was no methylation detected in any of the normal tissue samples.

Upregulated expression of miR-142 was induced by treatment with demethylation agents. An increase in the expression of miR-142 was observed in Saos-2 and MG63 cells treated with $15.55 \mathrm{nM}$ Aza, $1.5 \mathrm{nM}$ PBA and $15.55 \mathrm{nM} \mathrm{Aza}+1.5 \mathrm{nM}$ PBA (Fig. 2A). The miR-142 CpG island methylation status was examined using BSP following treatment with demeth- ylation agents. The results indicated that methylation was decreased by all the treatments, particularly by the combined Aza + PBA treatment (Fig. 2B).

Effects of demethylation agents on cell proliferation and invasion. CCK-8 was used to assess the effects of demethylation agents on Saos-2 and MG63 cell proliferation. It was observed that inhibition of proliferation was induced by Aza or PBA treatment alone, whereas a significantly higher level of inhibi- 
A

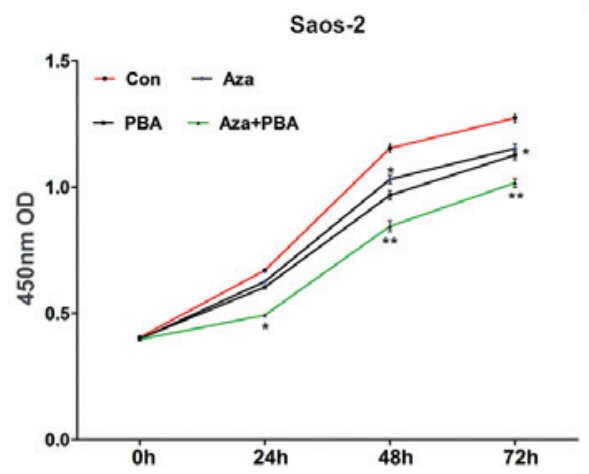

$\mathbf{C}$

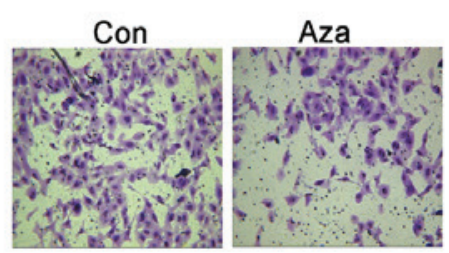

Saos-2

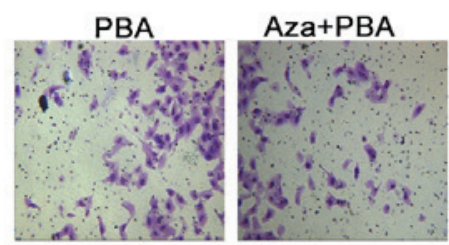

$\mathbf{E}$

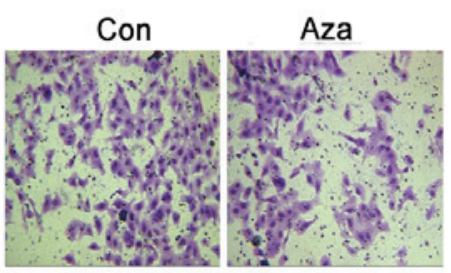

MG63

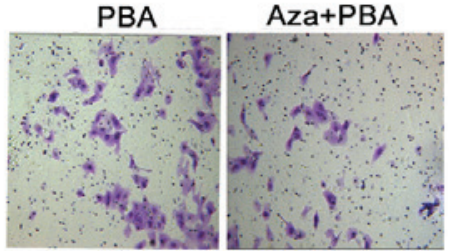

B

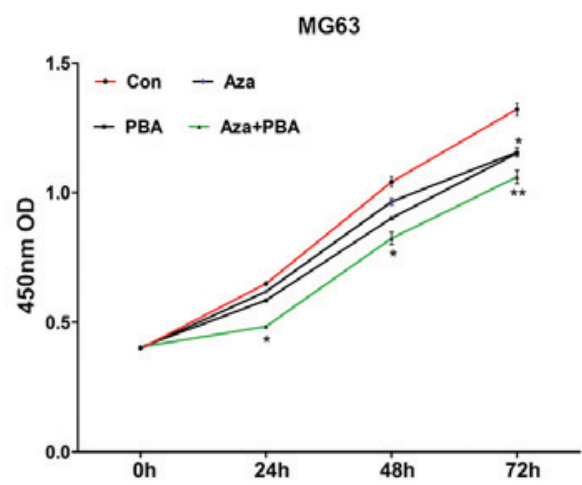

D

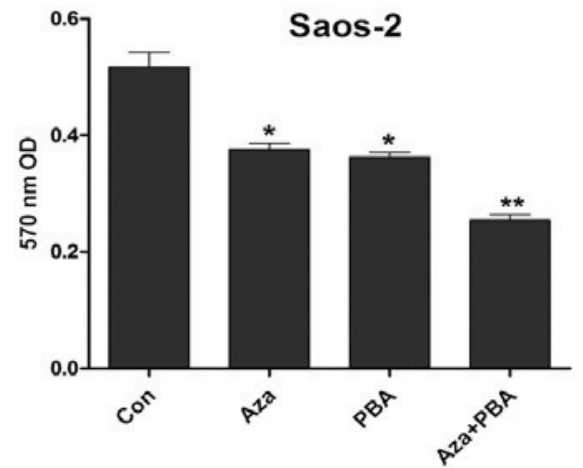

$\mathbf{F}$

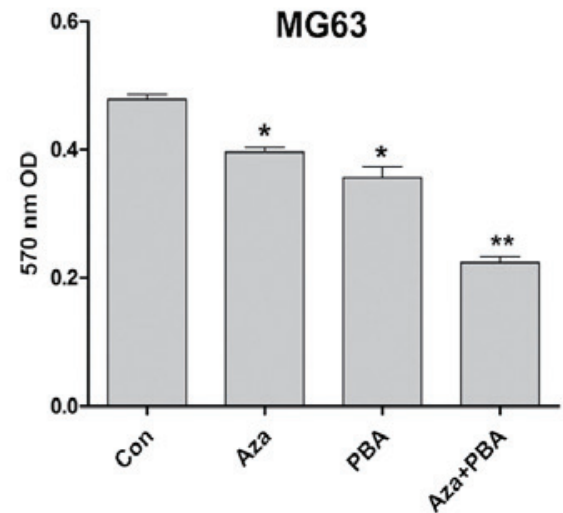

Figure 3. Effects of demethylation agents on cell proliferation and invasion. Cell proliferation was inhibited by demethylation agents in (A) Saos-2 and (B) MG63 cells. Cell invasive ability was reduced by demethylation agents in (C) Saos-2 and (E) MG63 cells. Quantification of the Transwell ${ }^{\mathrm{TM}}$ assay in (D) Saos-2 and (F) MG63 cells. Data are expressed as mean \pm standard deviation. "P $<0.05$ and ${ }^{* *} \mathrm{P}<0.01$ vs. control (Con). Aza, 5-Aza-2'-deoxycytidine; PBA, 4-phenylbutyric acid.

tion was observed in cells treated with Aza + PBA (Fig. 3A and B). The Transwell ${ }^{\mathrm{TM}}$ assay was used to measure the invasive ability of Saos-2 and MG63 cells following treatment with demethylation agents. The results indicated that their invasive ability was significantly decreased by all the treatments, particularly by the combined Aza + PBA treatment (Fig. 3C-F).

Demethylation agents induce the retardation of the $S$ phase in Saos-2 and MG63 cells. Flow cytometric analysis was used to analyze cell cycle alterations following treatment with demeth- ylation agents. In the cell cycle analysis, the ratio of cells in different phases of the cell cycle was as shown in Fig. 4A and $\mathrm{B}$. The results revealed that demethylation agents induced cell cycle arrest, thus inducing an increase in $\mathrm{G}_{2}$ phase cells, with a concomitant decrease in $\mathrm{S}$ phase cells (Fig. 4C).

\section{Discussion}

The survival rates of patients with OS have improved considerably following multiagent chemotherapy, with a 5-year survival 
A Saos-2
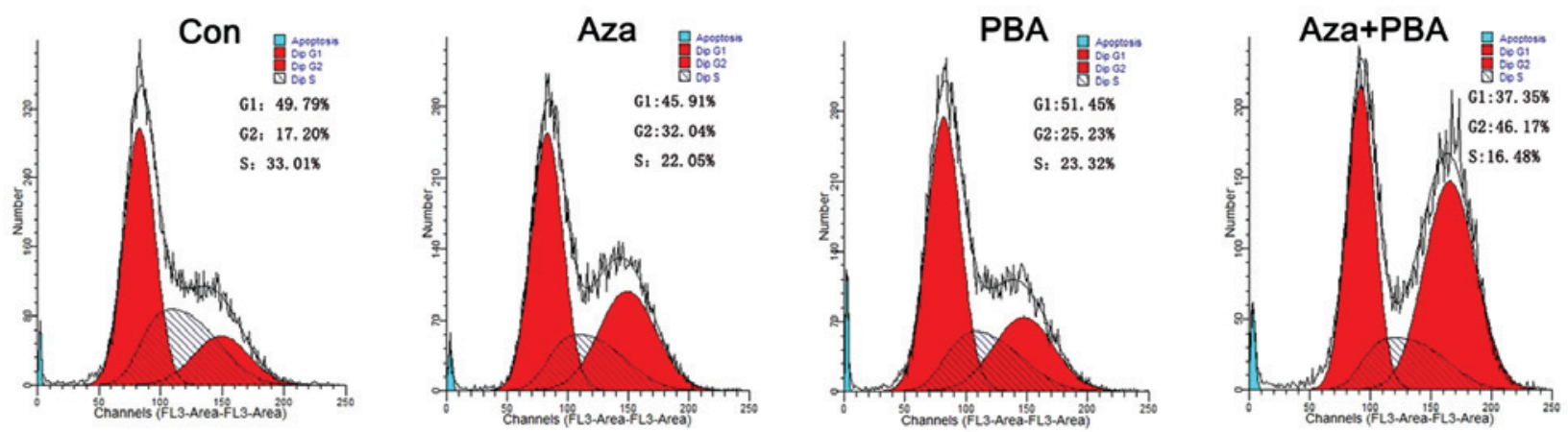

\section{B MG63}
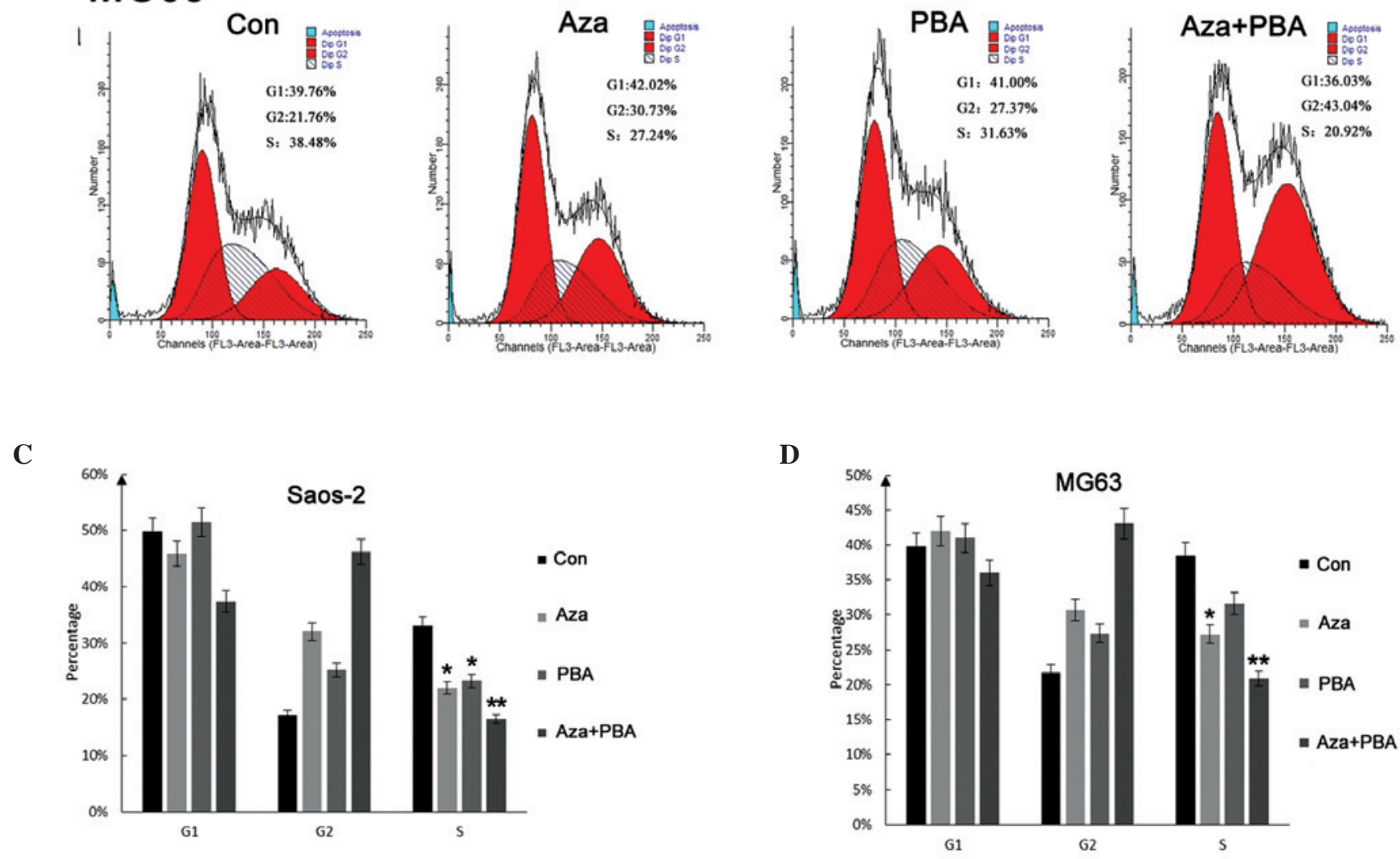

Figure 4. Cell cycle alterations following treatment with different demethylation agents in (A and C) Saos-2 and (B and D) MG63 cells. Data are expressed as mean \pm standard deviation. ${ }^{*} \mathrm{P}<0.05$ and ${ }^{* *} \mathrm{P}<0.01$ vs. control (Con). Aza, 5-Aza-2'-deoxycytidine; PBA, 4-phenylbutyric acid.

rate of $\sim 60 \%$ for patients without metastases (18). However, the survival rates have reached a plateau and novel biology-based therapies are imperative to achieve further improvement. At the molecular level, the majority of OSs exhibit complex genomic aberrations and highly variable patterns of gene expression. Emerging evidence has demonstrated that several miRNAs were altered in OS, some of which play a critical role in carcinogenesis $(6,10,19,20)$. Detailed understanding of miRNAs and their regulation mechanisms may aid the development of novel strategies to improve OS treatment.

The function of miR-142 has been primarily described in the hematopoietic and immune systems. Increased expression of miR-142 was observed in bronchioalveolar stem cells (BASCs), suggesting that miR-142 may play an important role in maintaining the self-renewal capacity of BASCs (15). In addition, ESCC patients with high expression levels of miR-142 exhibited poorer survival rates compared with those with low expression levels of miR-142 (16), whereas upregulated levels of miR-142 were involved in aggressive non-small-cell lung carcinoma (NSCLC) $(21,22)$. However, a recent study has suggested that miR-142 may be associated with the suppression of NSCLC cells (23). Wang et al (24) reported that miR-142 was upregulated by the NGX6 metastasis suppressor gene in colon cancer cells. Furthermore, their findings indicated that miR-142 plays a vital role in suppressing the proliferation of colon cancer cells and increasing the sensitivity of these cells to chemotherapeutic agents by targeting CD133, ABCG2 and Lgr5. It was also found that miR-142-3p acts as a tumor 
suppressor by targeting CD133, ABCG2 and Lgr5 in colon cancer cells (25).

In the present study, we confirmed that miR-142 was significantly decreased in OS tissues and cell lines. Although previous data demonstrated that miR-142 was downregulated in OS cell lines, as detected by global microarray analyses (17), the role of miR-142 in OS remains unknown.

Mature miRNAs are generated from primary miRNA transcripts (26), through an intermediate precursor (27). Increasing evidence indicates that, similar to protein-coding genes, epigenetic mechanisms, such as DNA methylation and histone acetylation, may be associated with the regulation of miRNA expression (28). DNA methylation at the dinucleotide $\mathrm{CpG}$ is one of the most common epigenetic modifications in eukaryotic genomes and plays a significant role in various biological processes (29). The majority of human miRNA genes have been found to be associated with CpG islands (30). However, the epigenetic mechanisms underlying the tissueand cell type-specific regulation of miR-142 have not been clearly determined in the majority of cases.

The present study revealed that a significantly higher level of miR-142 methylation was observed in OS compared with normal tissues. There was no methylation detected in any of the normal tissue samples. The findings suggested that the methylation mechanism of miR-142 may be involved in OS development. To investigate the involvement of epigenetic silencing, Saos- 2 and MG63 cells were initially treated with the demethylating agent Aza or/and PBA, which resulted in a concomitant increase of miR-142 transcripts, particularly in the group co-treated with Aza + PBA. To provide further evidence, BSP was used to demonstrate that the methylated CpG islands of miR-142 were significantly decreased by Aza and PBA treatment in Saos-2 and MG63 cells. Furthermore, a significant inhibition of proliferation in Saos-2 and MG63 cells by expression of miR-142 was induced by demethylation agents. It was also observed that increased miR-142 expression by Aza or PBA repressed cell migration. The overexpression of miR-142 has been shown to inhibit the proliferation and colony-forming ability of primitive hematopoietic cells (31). Some of these functions appear to depend on the cellular type and context, since it was reported that miR-142 acts as an anti-migratory factor in hepatocellular carcinoma cells (32) and has also been reported to be deregulated in mesenchymal tumors (33). In the present study, demethylation agents induced Saos-2 and MG63 cell cycle arrest, thus inducing an increase in $\mathrm{G}_{2}$ phase cells with a concomitant decrease in $\mathrm{S}$ phase cells. The exact mechanisms through which miR-142 inhibits cell proliferation and decreases $\mathrm{S}$ phase arrest remain unknown. One possible explanation is that multiple genes associated with the cell cycle are targeted by the miRNA signal. Therefore, other genes regulated by miR-142 that affect cell growth, the cell cycle and invasion should be further investigated.

In conclusion, the present study revealed that the expression level of miR-142 was significantly lower in OS tissues and cells due to hypermethylation. These findings suggested that miR-142 played an important role in the inhibition of cell proliferation and invasiveness of OS cell lines induced by demethylation agents. Of note, this study investigated the role of miR-142 only in vitro and the tumor-suppressive role of miR-142 in OS requires further confirmation by studies performed in vivo.

\section{References}

1. Kobayashi E, Hornicek FJ and Duan Z: MicroRNA involvement in osteosarcoma. Sarcoma 2012: 359739, 2012.

2. Cao ZQ, Shen Z and Huang WY: MicroRNA-802 promotes osteosarcoma cell proliferation by targeting p27. Asian Pac J Cancer Prev 14: 7081-7084, 2013.

3. Diao CY, Guo HB, Ouyang YR, et al: Screening for metastatic osteosarcoma biomarkers with a DNA microarray. Asian Pac J Cancer Prev 15: 1817-1822, 2014.

4. Benavente CA, McEvoy JD, Finkelstein D, et al: Cross-species genomic and epigenomic landscape of retinoblastoma. Oncotarget 4: 844-859, 2013.

5. Jin J, Cai L, Liu ZM and Zhou XS: miRNA-218 inhibits osteosarcoma cell migration and invasion by down-regulating of TIAM1, MMP2 and MMP9. Asian Pac J Cancer Prev 14: 3681-3684, 2013.

6. Maire G, Martin JW, Yoshimoto M, Chilton-MacNeill S, Zielenska M and Squire JA: Analysis of miRNA-gene expression-genomic profiles reveals complex mechanisms of microRNA deregulation in osteosarcoma. Cancer Genet 204: 138-146, 2011.

7. Lewis BP, Burge CB and Bartel DP: Conserved seed pairing, often flanked by adenosines, indicates that thousands of human genes are microRNA targets. Cell 120: 15-20, 2005.

8. Croce CM: Causes and consequences of microRNA dysregulation in cancer. Nat Rev Genet 10: 704-714, 2009.

9. Kelly TK, De Carvalho DD and Jones PA: Epigenetic modifications as therapeutic targets. Nat Biotechnol 28: 1069-1078, 2010.

10. Jones KB, Salah Z, Del Mare S, et al: miRNA signatures associate with pathogenesis and progression of osteosarcoma. Cancer Res 72: 1865-1877, 2012.

11. Poos K, Smida J, Nathrath M, Maugg D, Baumhoer D and Korsching E: How microRNA and transcription factor co-regulatory networks affect osteosarcoma cell proliferation. PLoS Comput Biol 9: e1003210, 2013.

12. Png KJ, Yoshida M, Zhang XH, et al: MicroRNA-335 inhibits tumor reinitiation and is silenced through genetic and epigenetic mechanisms in human breast cancer. Genes Dev 25: 226-231, 2011

13. Das S, Bryan K, Buckley PG, et al: Modulation of neuroblastoma disease pathogenesis by an extensive network of epigenetically regulated microRNAs. Oncogene 32: 2927-2936, 2013.

14. He C, Xiong J, Xu X, et al: Functional elucidation of MiR-34 in osteosarcoma cells and primary tumor samples. Biochem Biophys Res Commun 388: 35-40, 2009.

15. Qian S, Ding JY, Xie R, et al: MicroRNA expression profile of bronchioalveolar stem cells from mouse lung. Biochem Biophys Res Commun 377: 668-673, 2008.

16. Lin RJ, Xiao DW, Liao LD, et al: MiR-142-3p as a potential prognostic biomarker for esophageal squamous cell carcinoma. J Surg Oncol 105: 175-182, 2012 .

17. Namløs HM, Meza-Zepeda LA, Barøy T, et al: Modulation of the osteosarcoma expression phenotype by microRNAs. PLoS One 7: e48086, 2012

18. PosthumaDeBoer J, Witlox MA, Kaspers GJ and van Royen BJ: Molecular alterations as target for therapy in metastatic osteosarcoma: a review of literature. Clin Exp Metastasis 28: 493-503, 2011.

19. Baumhoer D, Zillmer S, Unger K, et al: MicroRNA profiling with correlation to gene expression revealed the oncogenic miR-17-92 cluster to be up-regulated in osteosarcoma. Cancer Genet 205: 212-219, 2012.

20. Lulla RR, Costa FF, Bischof JM, et al: Identification of differentially expressed microRNAs in osteosarcoma. Sarcoma 2011: 732690, 2011.

21. Boeri M, Verri C, Conte D, et al: MicroRNA signatures in tissues and plasma predict development and prognosis of computed tomography detected lung cancer. Proc Natl Acad Sci USA 108: 3713-3718, 2011.

22. Kaduthanam S, Gade S, Meister M, et al: Serum miR-142-3p is associated with early relapse in operable lung adenocarcinoma patients. Lung Cancer 80: 223-227, 2013.

23. Lei Z, Xu G, Wang L, et al: MiR-142-3p represses TGF- $\beta$-induced growth inhibition through repression of TGF $\beta R 1$ in non-small cell lung cancer. FASEB J 28: 2696-2704, 2014.

24. Wang XY, Wu MH, Liu F, et al: Differential miRNA expression and their target genes between NGX6-positive and negative colon cancer cells. Mol Cell Biochem 345: 283-290, 2010. 
25. Shen WW, Zeng Z, Zhu WX and Fu GH: MiR-142-3p functions as a tumor suppressor by targeting CD133, ABCG2, and Lgr5 in colon cancer cells. J Mol Med (Berl) 91: 989-1000, 2013.

26. Lee Y, Ahn C, Han J, et al: The nuclear RNase III Drosha initiates microRNA processing. Nature 425: 415-419, 2003.

27. Lee Y, Jeon K, Lee JT, Kim S and Kim VN: MicroRNA maturation: stepwise processing and subcellular localization. EMBO J 21: 4663-4670, 2002.

28. Vrba L, Garbe JC, Stampfer MR and Futscher BW: Epigenetic regulation of normal human mammary cell type-specific miRNAs. Genome Res 21: 2026-2037,2011.

29. Hackett JA and Surani MA: DNA methylation dynamics during the mammalian life cycle. Philos Trans R Soc Lond B Biol Sci 368: 20110328, 2013.
30. Weber B, Stresemann C, Brueckner B and Lyko F: Methylation of human microRNA genes in normal and neoplastic cells. Cell Cycle 6: 1001-1005, 2007.

31. Bissels U, Wild S, Tomiuk S, et al: Combined characterization of microRNA and mRNA profiles delineates early differentiation pathways of $\mathrm{CD}_{133^{+}}$and $\mathrm{CD} 34^{+}$hematopoietic stem and progenitor cells. Stem Cells 29: 847-857, 2011.

32. Wu L, Cai C, Wang X, Liu M, Li X and Tang $H$ : MicroRNA-142-3p, a new regulator of RAC1, suppresses the migration and invasion of hepatocellular carcinoma cells. FEBS Lett 585: 1322-1330, 2011.

33. Zhang P, Bill K, Liu J, et al: MiR-155 is a liposarcoma oncogene that targets casein kinase- $1 \alpha$ and enhances $\beta$-catenin signaling. Cancer Res 72: 1751-1762, 2012. 